\title{
JEKK
}

\section{Small Interfering RNA (siRNA) of Mini circle DNA Transfection (McD) is Encapsulated by Gold Nanoparticles from Folate's Receptors of Gold Conjugation as Curative Therapy for Non-Small's Cell of Lung Cancer}

\author{
I Gusti Ayu Stiti Sadvika*, I Gede Wikania Wira Wiguna*, Ni Putu Sri Indrani Remitha*, I Gede \\ Krisna Arim Sadeva*, Anak Agung Bagus Putra Indrakusuma*, Putri Ayu Wulandari", Ni Wayan \\ Sucindra Dewi ${ }^{* *}$, Agung Wiwiek Indrayani**
}

"Undergraduate Student, Faculty of Medicine, Udayana University, Bali, Indonesia, ${ }^{* *}$ Department of Pharmacology and Therapy, Faculty of Medicine, Udayana University, Bali, Indonesia

\begin{abstract}
Background: Lung cancer is one of the non-communicable diseases that have an increasing number of events that each year with a mortality rate of $18.4 \%$ and an incidence of $11.6 \%$. One of management in the NSCLC is currently in the form of surgery and adjuvant therapy. However, there were weaknesses and limitations in care for its patients therefore development of curative therapy for NSCLC's patients were needed. The aim of this review is to learn and investigate about the potential of siRNA that is transfected into $\mathrm{McD}$ and folate receptors alphaconjugated gold nanoparticle encapsulation as a therapeutic opportunity that could be developed as a treatment in management of NSCLC.

Method: The writing method used in this review article was the study method of literature. The used data comes from 45 of relevant literature sources and was arranged systematically in accordance with the topic of the problem discussed, along with the inclusion and exclusion criteria.

Result: This treatment could increase gene transfection, siRNA biodistribution in organs, reduced KRAS, Bcl-2, and VEGF expression. Moreover, siRNA had entered phase III clinical trials and FOLR1 antibodies were in phase II of clinical trials.

Conclusion: Utilization of siRNA specifically was designed for three genes such for KRAS, VEGF and Bcl-2 which were three genes that played a role in the pathogenesis of NSCLC, could be the right modality choice for treatment of NSCLC.
\end{abstract}

Keywords: NSCLC; small interfering RNA; gold nanoparticle; Minicircle DNA; FOLR1.

*Corresponding author, stiti.sadvika2210@gmail.com 


\section{Introduction}

Lung cancer is one of the noncommunicable diseases which occupies the top position as a cause of death with a percentage of $18.4 \%$ of the number of cancer cases worldwide (GLOBOCAN, 2018). Based on research by De Groot et al (2012), lung cancer is dominated by men over the age of 50 years. Judging from the cause, as many as $80 \%$ of lung cancer's deaths are caused by smoking. In fact, according to the US Centers for Disease Control and Prevention, exposure to Other People's Cigarette Smoke (AROL) causes 50,000 deaths each year. Moreover, the population of Indonesians who smoke is increasing. Based on data from Tobacco Atlas (2012), the number of cigarettes consumed in Indonesia increased from 182 billion sticks to 260.8 billion. In addition to smoking, unhealthy lifestyles such as alcohol consumption and genetic factors also contribute to increasing the risk of lung cancer. ${ }^{34}$

One type of lung cancer that accounts for more than $80 \%$ of all lung cancer cases is Non Small-cell Lung Carcinoma (NSCLC). The management of NSCLC therefore far depends on the condition of the patient's prognosis and staging. Patients who are in stage I, II, and IIIA generally undergo surgical procedures to remove the tumor. However, this can still cause recurrence in patients suffering from NSCLC type of adenocarcinoma with a greater mutation fraction and lead to a tendency for earlier metastases $^{45}$. Besides surgery, there are adjuvant therapies, such as radiotherapy, chemotherapy, and target therapy. Radiotherapy is a therapeutic method used to reduce the size of the tumor, but the therapeutic efficacy is less than optimal because it is limited by systemic tumor progression, the presence of local or distant metastases, and tumor resistance itself. ${ }^{16}$ Similar to radiotherapy, chemotherapy also has high toxicity and side effects.

The level of expression of KRAS, Bcl-2, and VEGF genes is a gene that can indicate the prognosis in NSCLC patients so that it becomes a biomarker that can be used as NSCLC curative therapy. The mutated KRAS activation becomes one of the signaling pathways that play a role in resistance to EGFR tyrosine kinase inhibitors (TKIs), as well as monoclonal antibodies ${ }^{4}$. High expression of Bcl-2 can prevent cancer cells from apoptosis. ${ }^{28}$ The VEGF plays a role in the progression of cancer through angiogenesis by stimulating vascular permeability of blood vessels and increasing extravasation of plasma proteins. ${ }^{14}$

Small interfering RNA (siRNA) is part of RNAi (RNA interference) which has the ability to regulate gene expression, primarily in performing its function as gene silencing of specific molecules. siRNA will be designed specifically for the KRAS, Bcl-2, and VEGF genes as advanced curative therapy in NSCLC patients. However, challenges in administering siRNA therapy include instability in blood serum, accumulation in nonspecific tissue, and inefficient intracellular release ${ }^{25}$.Therefore an effective, safe, and capable carrier that increases the concentration of siRNA, is needed in the distribution of siRNA to the target cell so as to produce maximum efficacy. ${ }^{7}$

$\mathrm{McD}$ technology (DNA minicircle) is a nonviral based vector that can be used in siRNA transfection. DNA minicircle is able to maintain the expression of genes carried 10-1000 times higher compared to conventional plasmids. However, gene transfection via $\mathrm{McD}$ is not single selective to the desired target cell. Therefore, McD-based siRNA administration will be encapsulated by gold nanoparticles (AuNP). ${ }^{23}$

Gold nanoparticle (AuNP) is an encapsulation based on nanoparticle technology that can be used as a carrier of substances aimed at target cells. However, the distribution of siRNA by encapsulation of AuNP requires a specific ligand to facilitate siRNA to target the desired cell. Thus, AuNP will be conjugated with the alpha receptor Folate (FOLR1) antibody to form AuNP-FOLR1. FOLR1 is a protein that functions as a folate transporter and is overexpressed in lung cancer. The FOLR1 antibody binds to the folate receptor and creates a ligand receptor complex that will later regulate signaling in cancer. ${ }^{2}$

\section{Methods}

The method that is used in writing this literature review is a literature review method where the data used comes from various 
relevant literature sources obtained from several search engines such as Pubmed, NCBI and Google Scholar. The literature obtained is then filtered based on inclusion and exclusion criteria. Inclusion criteria include journals discussing points on keywords, are research journals and article reviews and have a maximum time span of journals of the past ten years. While exclusion criteria include not containing information according to keywords, journals are protocol only articles and have a range of more than ten years. Furthermore, from all selected journals, 45 journals were found that fit the inclusion and exclusion criteria. The data obtained are in the form of quantitative and qualitative data which are then compiled systematically and in accordance with each topic discussed so that there are groups that represent the entire contents of the writing.

\section{Result}

Specific SiRNA silencing of KRAS, $\mathrm{Bcl}-2$, and VEGF genes will be delivered by DNA minicircle vectors with significant gene transfection. Chabot, et al (2012) in their study used the same number of copies for each experiment. The level of gene expression was analyzed with green-fluorescent protein (GFP). $\mathrm{McD}$ (MC-GFP) and conventional plasmid (pGFP) construction are injected into the tibial cranial mouse. Mice were injected with MCGFP or p-GFP and Co-transfection TurboFP635 plasmids as internal controls. The results of the MC-GFP GFP fluorescence analysis in all muscle samples were 2.4-10.8 times higher than the p-GFP. The fluorescence ratio of GFP and internal control (TurboFP635 shows that MCGFP causes gene expression 2.5-4 times higher than p-GFP (2.6 times \pm 0.5 increase on day 8 and 4 times increase \pm 0.9 on day 18$)^{3}$.

Research in vivo Reuveni et al, (2011) regarding comparisons between actively designed AuNP biodistribution with conjugation of EGFR antibodies, passively with anti-immunoglubulin $\mathrm{G}$ conjugation and six hours post administration without conjugation as control. The results showed a significant increase in organ biodistribution in AuNP conjugated EGFR antibodies compared to the other two groups in tumor tissue. In line with this, Takeuchi, et al (2018) research on PEG conjugated AuNP biodistribution. Results show that PEG conjugated AuNP is significantly higher in the lungs starting from six hours postinjection and increasing organ accumulation for up to 48 hours. ${ }^{37}$

At 24, 48 and 96 hours after siRNA nanoparticle injection, cell lysate was prepared for analysis using qPCR and Western blot. The level of KRAS silencing was assessed in all cells by immunobloting techniques. The results of measurement of mRNA level with qPCR showed a significant decrease in KRAS expression starting from the analysis in the first 24 hours with a constant expression level of up to 96 hours. In vivo studies by Pecot, et al (2014) showed a decrease in KRAS expression using KRAS siRNA and NC siRNA constructs as controls administered intravenously in rats for four weeks showed a reduction in luminescence by $81 \%$ compared to control siRNA $(\mathrm{P}<0.01)$ $(\mathrm{P}<0.01)(\mathrm{P}<0.01){ }^{27}$

In vivo research by Tekedereli et al. (2013) to determine the effect of silencing the Bcl-2 gene in mice with MDA-MB-231 tumor cells in orthotopic xenograft models was carried out by NL-Control-siRNA and NL-Bcl-2siRNA administration at a dose of 0.075 and $0.15 \mathrm{mg}$ siRNA / $\mathrm{kgBB}$ IV via the tail vein. Tumors were removed on the 2 nd, 4 th and 6 th day after injection and analyzed with densitometry analysis bands. Significantly decreased $\mathrm{Bcl}-2$ protein expression using NLBcl-2-siRNA compared to NL-Control-siRNA at the same dose. ${ }^{38}$

Research Yuan et al. (2015) to determine the effect of VEGF silencing with siRNA in vivo. C57BL / 6 mice were injected with Lewis Lung Carcinoma (LLC) subcutaneously. When the tumor reached $\pm 0.5 \mathrm{~cm}$ in diameter, samples were injected with $\mathrm{OH}$-antitumoral control, $\mathrm{OH}$ VEGF, ppp-control or ppp-VEGF at a dose of $20 \mu \mathrm{g}$ for each injection twice a week for two weeks of administration. After two migraines it was found that the number of blood vessels due to VEGF induced angiogenesis in LLC showed a significant reduction with OH-VEGF and pppVEGF injections compared to the control group. ${ }^{43}$ 


\section{Discussion}

Lung cancer cells have an increased proliferation activity associated with the KRAS gene (Rini, et al, 2011). The KRAS gene (Kirsten Rat Sarcoma) which encodes the RAS protein, binds to the guanosine nucleotides, namely guanosin diphosphate (GDP) and guanosin triphosphate (GTP). RAS protein will be activated by transforming GDP into GTP Furthermore, inactivation of RAS protein occurs through the activity of the GTPase enzyme that hydrolyzes GTP to GDP. ${ }^{19}$

Intrinsic pathway apoptosis is induced by mitochondrial stress resulting in the release of proapoptotic factors such as cytochrome c and AIF from mitochondrial intermembrane (Wong, 2011). The release was regulated by the Bcl-2 family, namely BAX and BAD (Sari, 2018). Cytochrome c released into the cytoplasm binds to Apaf-1 and forms the Caspase Recruitment Domain (CARD). The CARD compound forms an apoptosome complex that activates the initiator caspase, caspase-9, which in turn activates the executor caspase by converting procaspase- 3 to caspase3. In NSCLC lung cancer, excessive expression occurs in Bcl-2 so that cancer cells are able to overcome the apoptotic signals from Myc and RAS. ${ }^{33,41}$

In its development, the pathogenesis of cancer is related to angiogenesis. Angiogenesis is initiated by vascular endothelial growth factor (VEGF). ${ }^{6,21}$ One mechanism that is able to regulate VEGF expression is hypoxia. Hypoxia inducible factor-1 (HIF-1) is the most important mediator of the hypoxic response. ${ }^{13}$ Cytokines and growth factors are also able to regulate the expression of angiogenic factors in cancer cells so that it will indirectly stimulate angiogenesis, such as HER2 and EGFR, COX -2 and plateletderived growth factors (PDGFs). ${ }^{42}$

SiRNA therapy is a new anticancer approach that has undergone phase III clinical trial involving \pm 6500 cancer patients. ${ }^{5}$ SiRNAbased therapy has potential as a cancer therapy, including NSCLC lung cancer. SiRNA therapy also has several advantages over traditional pharmaceutical drugs. Moreover, siRNA is a form of RNA interference (RNAi) which is a post-transcription gene silencing mechanism. The work of siRNA is done by triggering the degradation of mRNA by attaching doublestranded RNA (dsRNA) into the target cell. RNAi is able to inhibit the expression of genes contained in specific sequences through the termination of mRNA that has a homologous short sequence. ${ }^{29}$

Gene silencing therapy method with siRNA is a promising method. However, siRNA has a weakness, namely the instability of siRNA molecules while in the cell due to the activity of nuclease enzymes which break down the structure of siRNA into its nucleotide bases, molecules that are hydrophilic so that they are easily degraded, molecules that will undergo endosomal breakdown by liposomes, molecules that must enter the cell's nucleus to carry out its functions, and the structure of the DNA and DNA are folded and protected so that siRNA cannot function optimally as gene silencingbased therapy. The mechanism of action of siRNA is at the stage of transcription and posttranscription of the protein by binding specifically to the target mRNA so that it causes downregulation, even silencing targeted genes. ${ }^{11}$

Minicircle DNA (McD) is a nonviral transfection vector derived from minicircle producer plasmids (pMC). Plasmids are extrachromosomal DNA found in living cells and are able to replicate autonomously. ${ }^{23} \mathrm{McD}$ production comes from a special parental plasmid strain of E. coli which is engineered to cause the parent plasmid to breed and produce minicircles. McD is produced conditionally by the expression of the aseC 31 integrase induced by intramolecular recombination (cis-). Then, $\mathrm{McD}$ was planted in a special strain of E. coli host bacteria with an arabinose-induced system to express integrase $31 \mathrm{C} 31$ and I-SceI endonuclease simultaneously. $\mathrm{McD}$ is a vector that is easier to produce, inexpensive, stable, and safer than ordinary plasmid methods. McD's advantage over conventional plasmids is that it can increase transfection because of its smaller size. McD does not contain inflammatory sequences, sequences of replication sources, is free of expression of viral antigens, does not contain antibiotic resistance markers or bacteria from replication so they have a high level of security in the body. $\mathrm{McD}$ can increase 
transgene expression 10-1000 times higher than conventional DNA plasmids. ${ }^{1}$

Gold nanoparticles (AuNP) are nanoparticles with different properties from inert gold in nature and are synthesized from natural materials, biomaterial egg shells and sunlight. ${ }^{9}$ AuNP is nano-sized $(1 \mathrm{~nm}-8 \mu \mathrm{m})$ so that it is able to cross cell membranes and nuclei to interact with DNA, shows excellent penetration into cells, can increase cellular absorption efficiency and delivery to reach target tissue. As a cancer therapy, AuNP offers a nano platform material for ultrasensitive detection of biomolecules, killing cancer cells with hyperthermal treatment, labeling cells and proteins, easily conjugated so as to suppress side effects, and able to deliver therapeutic agents in cells. ${ }^{24}$ Another advantage of AuNP is its high surface area so it is easily modified for therapies that target specific genes or cells, are noncytotoxic, safe, environmentally friendly, increase half life, and are biocompatible with the body. ${ }^{15}$ AuNP can deliver hydrophilic and hydrophobic drugs because of its characteristic that monolayers are able to change the structural properties of lipid molecules that interact directly with the surface of nanoparticles (Lan, et al., 2013). Later, AuNP will be conjugated with the FOLR1 (Folate Receptor alpha) antibody to make specific drugs work on targets related to NSCLC lung cancer pathogenesis.

Folate receptor alpha (FOLR1) is a membrane protein receptor that is bound to glycosylphosphatidylinositol (GPI) and has high expression in lung cancer. FOLR1 shows very low or even non-expression on normal cells. FOLR 1 can be translocated to the nucleus and act as a transcription factor and binds to the cis-regulator element. Through this mechanism, FOLR1 can directly regulate the expression of key development genes in cancer cells. FOLR1 has also been shown to inhibit caveolin-1 so that it supports the growth and proliferation of tumor cells that are not dependent on the port and increase the development of cancer. The presence of anti-FOLR1 as a target for cancer therapy has been widely developed and has entered a phase II clinical trial. ${ }^{2}$

McD's construction begins with the preparation of plasmid minicircles made by inserting DNA elements: KanR genes which are enclosed in the attB and attP binding sites of the 31C31 integrase bacteriophage, as well as endonuclease A fragments amplified using PCR to form the pMC.HFIX or pMC.RSV.HAAT binding site. ZYCY10P3S2T strain will be added. The PCR primers used were 5'TCCTGTTACCAGTGGCTGCT and 5'AGTTCGGTGTAGGTCGTTCG. After that, bacterial amplification was carried out by combining $100 \mu \mathrm{l}$ of culture for every $400 \mathrm{ml}$ of TB containing Kan $(50 \mu \mathrm{g} / \mathrm{ml})$ and continued incubation for 16-18 hours with a $\mathrm{pH}$ of $\sim 6.5$. The mixture is combined with culture and used to isolate $\mathrm{McD}$ vectors using a plasmid purification kit. $^{23}$

SiRNA construction is carried out using PCR with the basic principle of cutting short hairpin RNA (shRNA) from a plasmid vector into 2 short RNA chains that are complementary to each other. First the KRAS sequence is incorporated in a siRNA encoding DNA template consisting of a double-stranded class III $\mathrm{T} 7$ promoter then transcribed with R7 polymerase $\mathrm{T} 7$ to produce a single chain RNA transcript containing the target sequence of the KRAS gene. Transcripts were incubated to form the structure of the hairpin shRNA, then cut using the enzyme dicon RNA endonuclease. The process is then repeated using VEGF and Bcl-2 sequences. The siRNA transfection method is using McD vectors which are cut with endonuclease restriction enzymes, then KRAS, VEGF, and Bcl-2 specific siRNAs are inserted into $\mathrm{McD}$, and the bonds are strengthened with ligase enzymes. ${ }^{26}$

AuNP is formed through the green biosynthesis method which uses natural biomaterial eggshell (ESM) membrane. This method is done by soaking $\mathrm{HAuCl} 4$ solution without using a reducing agent (Epanchintseva, 2019). Next, AuNP is stabilized using heterobifunctional PEG with thiol groups at one end and one of the carboxyl (HS-EG (8) - $\mathrm{CH} 2$ ) 2-COOH, PEG-COOH) or azide (HS- $(\mathrm{CH} 2) 3-$ CONH-EG groups (6) - (CH2) 2-N3, PEG-N3) on the other hand, then incubated for 16 hours, and purified by centrifugation. The conjugation of the isolated siRNA chain was removed through incubation of AuNP (5nm) with the siRNA chain in the DEPC and SDS $0.028 \%$ then a $0.1 \mathrm{M} \mathrm{NaCl}$ concentration was added and then 
quantified with fluorescence using a GelRed intercalator. ${ }^{35}$

FOLR1-conjugated gold nanoparticles can be synthesized with a linker such as glutathione as a stabilizer and an agent for coupling. There are steps proposed in making this conjugation, namely glutathione that binds to the surface of AuNP through cysteine groups. Then, the carboxyl group from glutathione will be activated through N, Ndicyclochexylcarbodiimide (DCC) and Nhydroxysuccin-imide (NHS). Finally, folate / glutathione / AuNP is formed through the reaction between the carboxyl glutathione group and the amine group of folic acid..$^{32,45}$

Administrative modalities will be given intravenously (IV) because they provide direct access to blood circulation by injection and can be spread easily into the tissues. ${ }^{40}$ In addition, IV administration of gold nanoparticles has a lower toxicity content compared to other administrative channels.

The modality administered in IV causes the modality not to pass through absorption and directly circulates in the blood vessels. Furthermore, the modality will be distributed to the organs of the body. The modality of siRNA encapsulation AuNP reaches peak accumulation in organs \pm 4-12 hours after injection and accumulates constantly after 24 hours (Takeuchi et al, 2018). Accumulation is directly proportional to the increased elimination of AuNP through urine so that there is no deposition in organs. PEG conjugated AuNP causes modalities to be undetectable by the immune system to avoid endocystosis and siRNA degradation that is transfected through $\mathrm{McD}$ as it passes through the endosomal pathway. ${ }^{37} \mathrm{McD}$-siRNA will go to the nucleus to replicate and carry out its function. AuNP which has brought McD-siRNA to target cells is excreted via exocytosis to be re-channeled into blood vessels and accumulated in the kidneys after six hours after injection to be excreted in urine. McD-siRNA will continue to be expressed until the expression of the target gene namely KRAS, Bcl-2 and VEGF is lost from the target cell. Once the gene expression is stopped, siRNA will be naturally degraded by the body. ${ }^{17}$

The therapeutic effect of siRNA is seen through a decrease in downstream signals and inhibition of NSCLC lung cancer cell proliferation. Research by Pecot et al. 2014 shows that siRNA is able to silence the KRAS gene. KRAS is a gene that encodes the function of cell proliferation and express RAS proteins. The siRNA molecule is able to inactivate the RAS protein by reducing the affinity of the picomolar GTPase binding and cellular concentration of GTP so that the binding process can be inhibited. This causes the hydrolysis of GDP to GTP can also be stopped. ${ }^{27}$

The results of the study by Geng, et al., 2013 showed that siRNA was able to induce apoptosis by increasing the ratio of Bax / Bcl-2 and at the same time silencing Bcl-2. Increased expression of $\mathrm{BAX}$ protein which is proapoptosis causes impaired permeability resulting in cytochrome $\mathrm{c}$ and AIF release from mitochondrial intermembrane. Cytochrome c released into the cytoplasm will bind to Apaf-1 and form CARD. Combined several CARDs then form an apoptosome complex that will activate the initiator caspase, namely caspase-9 so that caspase-3 activation occurs as the executor caspase. ${ }^{33}$

Research Jafari, et al., (2016) showed that siRNA was able to inhibit the expression of VEGF and HIF- $\alpha$ factors so as to prevent angiogenesis by decreasing the regulation of molecules involved in angiogenesis, such as: neurophilin, angiogenin, interleukin 6 and 8, MCP-1 and TGF- $\beta$. The mechanism of inhibition of VEGF expression, including VEGF-A, is initiated by activating PPAR $\gamma$ which then inactivates the phosphatidylinositol 3-kinase / protein kinase B (AKT) pathway. The unactivated phosphatidylinositol 3-kinase / protein kinase B (AKT) pathway will result in decreased transcriptional mRNA transcription and expression of VEGF-A resulting in decreased VEGFR-1 expression leading to decreased angiogenesis. ${ }^{18}$

\section{Conclusion}

The modality construction mechanism starts from making $\mathrm{McD}$, then constructing siRNA using PCR, inserting specific KRAS, VEGF, and Bcl-2 siRNA into McD, making AuNP through the green biosynthesis method, and 
continuing with conjugation of FOLR1 to AuNP. Modality administration is given intravenously (IV) because it gives direct access to blood circulation. After doing its work, AuNP will be excreted via exocytosis, while McDsiRNA will continue to be expressed until the expression of the target gene, KRAS, Bcl-2 and VEGF, is lost from the target cell. The mechanism of action of the modality as NSCLC management, namely inhibiting cell proliferation by silencing the KRAS gene, inducing apoptosis by increasing the $\mathrm{Bax} / \mathrm{Bcl}-$ 2 ratio and simultaneously silencing $\mathrm{Bcl}-2$, as well as inhibiting the expression of VEGF and HIF- $\alpha$ factors so as to prevent angiogenesis. The clinical effect of the modality is to increase gene transfection, increase the biodistribution of siRNA in organs, decrease the expression of KRAS, Bcl-2, and VEGF. Moreover, siRNA has entered clinical trials up to phase III and the FOLR1 antibody is in phase II clinical trials.

\section{Acknowledgement}

Our thanks to JEKK for allowing us to modify templates they had developed.

\section{References}

1. Alto, P. 2017. Mini Circle DNA Technology. System Biosciences. 7:1-30.

2. Cheung, A., Bax, H.J., Josephs, D.H., Ilieva, K.M., Pellizari, G., Opzoomer, J., Bloomfield, J. 2016. Taegeting Folate Receptor Alpha for Cancer Treatment. Oncotarget. 7(32):52553-52569.

3. Chabot, S., Orio, J., Schmeer, M., Schleef, M., Golzio, M., Teissie, J. 2012. Minicircle DNA Electrotransfer for Efficient TissueTargeted Gene Delivery. Gene Therapy.1-7.

4. Chong, C.R., Janne, P.A. 2013.The Quest to Overcome Resistance to EGFR-Targeted Therapies in Cancer. Nat Med.19(11):1389400.
5. Chakraborty, C., Sharma, A.R., Sharma, G., Doss, C., \& Lee, S.S. 2017. Therapeutic miRNA and siRNA, Moving from Bench to Clinic as Next Generation Medicine. Molecular therapy. Nucleic acids. 8:132143.

6. Chen, P., Cheng, H., Wang, J., Wang, S. 2014. Prostate Cancer-Derived CCN3 Induces M2 Macrophage Infiltration and Contributes to Angiogenesis in Prostate Cancer Microenvironment. Oncotarget. 5(6):1595-608.

7. Dana, H., Chalbatani, G.M., Mahmoodzadeh, H., Karimloo, R., Rezaiean, O., Moradzadeh, A. 2017. Molecular Mechanisms and Biological Functions of siRNA. International Journal of Biomedical Science.13(2):48-53.

8. De Groot, P., Reginald, F., Munden. 2012. Lung Cancer Epidemiology, Risk Factors, and Prevention. Radiol Clin N Am Elsevier:863-876.

9. Deb, S., Patra, H.K., Lahiri, P., Dasgupta, A.K., Chakrabarti, K., Chaudhuri, U. 2011. Multistability in Platelets and Their Response to Gold Nanoparticles. Nanomed: Nanotechnol Biol Med. 7:376-384.

10. Epanchintseva, A.,S., Poletaeva, J.,E., Pyshnyi, D.,V., Ryabchikova, E.,I., Pyshnaya, I.,A. 2019. Long-Term Stability and Scale-Up of Noncovalently Bound Gold Nanoparticle-siRNA Suspensions. Beilstein Journal of Nanotechnology. 10:2568-2578.

11. Fakhr, E., Zare, F., Teimoori-Toolabi, L. 2016. Precise and Efficient siRNA Design, A Key Point in Competent Gene Silencing. Cancer Gene Therapy:1-10.

12. Ferlay, J., Colombet, M., Soerjomataram, I., Mathers, C., Parkin, D.M., Piñeros, 
M., Znaor, A., Bray, F. 2018. Estimating the Global Cancer Incidence and Mortality in 2018: GLOBOCAN Sources and Methods. International Journal of Cancer. 144(8):1941-1953.

13. Figg, K.M.C. 2011. Angiogenesis Inhibitors-Current Strategies and Future Prospects. 60:222-243.

14. Frezzetti, D., Gallo, M., Maiello, M.R., D'Alessio, A., Esposito, C., Chicchinelli, N. 2017. VEFG as Potential Target in Lung Cancer. Expert Opinion on Therapeutic Targets. 21(10):959-966.

15. Guo, Q., Yuan, J., Zeng, J. 2014. Biosynthesis of Gold Nanoparticles Using a Kind of Flavonol: Dihydromyricetin Colloids and Surfaces A: Physicochemical and Engineering Aspects. 441:127-132.

16. Huang, C., Ju, D., Chang, C., Reddy, P.M., Velmurugan, B.K. 2017. A Review on The Effects of Current Chemotherapy Drugs Natural Agents in Treating Non-Small Cell Lung Cancer. Biomedicine. 7(4):12-23.

17. Huang, Y., Cheng, Q., Ji, J., Zheng, S., Du, L., Meng, L., Wu, Y., Zhao, D., Wang, X., Lai, L., Cao, H., Xiao, K., Gao, S., Liang, Z. 2016. Pharmacokinetic Behaviors of Intravenously Administered siRNA in Glandular Tissue. Theranostic. 6(10):15281541.

18. Jafari, S.M., Yazdi, F., Masoomi, K.M., Alizadeh, J., Rahmati, M., Zarei, M.A. 2016. The siRNA-Mediated Down-Regulation of Vascular. Marranci. 10(6): 316-26.

19. Lewandowska, M.A. 2013. KRAS and BRAF Mutation Analysis in Colorectal Adenocarcinoma Specimens with a Low Percentage of Tumor Cells. Original Research Article. 17:193-203.
20. Lee, T., Jeong, E., Min, I.J., Kim, S.Y., Kim, H.R., Kim, C.H. 2017. Altered Expression of Cellular Proliferation, Apoptosis and The Cell Cycle-Related Genes in Lung Cancer Cells with Acquired Resistance to EGFR Tyrosine Kinase Inhibitors. Oncol Lett. 14:2191-7.

21. Lin, S.A.M.H., Luidens, M.K., Davis, P.J. 2014. Modulation of Angiogenesis by Thyroid Hormone and Hormone Analogues, Implications for Cancer Management. Angiogenesis:1-7.

22. Lan, M.Y., Hsu, Y.B., Hsu, C.H., Ho, C.Y., Lin, J.C., Lee, S.W. 2013. Induction of Apoptosis by High-Dose Gold Nanoparticles in Nasopharyngeal Carcinoma Cells. Auris Nasus Larynx. 40:563-568.

23. Maniar, L.E.G., Maniar, J.M., Chen, Z., Lu, J., Fire, A.Z., Kay, M.A. 2013. Minicircle DNA Vectors Achieve Sustained Expression Reflected by Active Chromatin and Transcriptional Level. Molecular Therapy. 21(1):131-138.

24. Mishra, A., Tripathy, S.K., Yun, S.I. 2012. Fungus Mediated Synthesis of Gold Nanoparticles and Their Conjugation with Genomic DNA Isolated from Escherichia coli and Staphylococcus aureus. Process Biochem. 47:701-711.

25. Min, S.S., Young, J.K. 2010. Efficient and Targeted Delivery of siRNA In Vivo. FEBS Journal:4814-4827.

26. Geng, Z., Zhang, M., Pan, X., Wang L. 2013. Bcl-2 Gene Silencing by RNA Interference Inhibits The Growth of The Human Gallbladder Carcinoma Cell Line 
GBC-SD In Vitro and In Vivo. Oncology Reports. 30:793-800.

27. Pecot, C.V., Wu, S.Y., Bellister, S., Filant, J., Rupaimoole, R., Hisamatsu, T., Nagaraja, A.S. 2014. Therapeutic Silencing of KRAS Using Systemically Delivered SiRNAs. Molecular Cancer Therapeutics. 13(12):2876-2885.

28. Ping-Li, S., Hironobu, S., Hongwen, G. 2017. Bcl-2 Family in Non-Small Cell Lung Cancer: Its Prognostic and Therapeutic Implications. Pathology International:1-10.

29. Rahman, M.M., McFadden, G. 2011. Modulation of NF- $\kappa B$ Signalling by Microbial Pathogens. NIH Public Access. 9(4):291-306.

30. Reuveni, T., Motiei, M., Romman, Z., Popovtzer, A., Popovtzer, R. 2011. Targeted Gold Nanoparticles Enable Molecular CT Imaging of Cancer: An In Vivo Study. International Journal of Nanomedicine. 6:2859-2864.

31. Rini, C., Widjajanto, E., Loekito, R. 2011. Peranan Curcumin terhadap Proliferasi, Apoptosis dan Diferensiasi Hepatosit Mice Balb / C yang Dipapar dengan Benzapyrene. JExp Life Sci. 1(2):64-71.

32. Samadian, dkk. 2016. Folate-Conjugated Gold Nanoparticle as a New Nanopaltform for Targeted Cancer Therapy. J Cancer Res Clin Oncol. 142(11):2217-2229.

33. Sari, L.M. 2018. Apoptosis, Mekanisme Molekuler Kematian Sel. Cakradonya Dent J. 10(2): 65-70.

34. Sholih, M.G., Perwitasari, D.A., Hendriani, R., Sukandar, H., Barliana, I., Suwantika, A. et.al. 2019. Risk Factor of Lung Cancer in Indonesia. 2019 Journal of Advanced
Pharmacy Education \& Research. 9(2): 4145.

35. Son, S., Kim, N., You, D.,G., Yoon, H.,Y., dkk. 2017. Antitumor Therapeutic Application of Self-Assembled RNAiAuNP Nanoconstructs: Combination of VEGF-RNAi and Photothermal Ablation. Theranostic. 7(1): 9-22.

36. Takeuchi, I., Nobata, S., Oiri, N., Tomoda, K., Makino, K. 2017. Biodistribution and Excretion of Colloidal Gold Nanoparticles After Intravenous Injection: Effects of Particle Size. Bio-Medical Materials and Engineering. 28: 315-323.

37. Takeuchi, I., Onaka, H., Makino, K. 2018. Biodistribution of Colloidal Gold Nanoparticles After Intravenous Injection: Effects of PEGylation at The Same Particle Size. Bio-Medical Materials and Engineering. 29:205-215.

38. Tekedereli, I., Alpay, S.N., Akar, U., Yuca, E., Ayugo-Rodriguez, C., Han, H., Sood, A.K., Lopez-Berestein, G., Ozpolat, B. 2013. Therapeutic Silencing of Bcl-2 by Systemically Administered siRNA Nanotherapeutics Inhibits Tumor Growth by Autophagy and Apoptosis and Enhances the Efficacy of Chemotherapy in Orthotopic Xenograft Models of ER (-) and ER (+) Breast Cancer. Molecular TherapyNucleic Acids. 2(121):2162-2531.

39. Tobacco Control Support Centre-IAKMI, Kementerian Kesehatan. 2012. Bunga Rampai Fakta Tembakau dan Permasalahannya di Indonesia Tahun 2012. Jakarta: Tobacco Control Support CentreIAKMI.

40. Verma, P.,Thakur, A.S., Deshmukh, K., Jha A.K., Verma, S. 2010. Routes of Drug 
Administration. International Journal of Pharmaceutical Studies and Research. 1(1):54-59.

41. Wong, R.S.Y. 2011. Apoptosis in Cancer, From Pathogenesis to Treatment. J Exp Clin Cancer Res. 30(1):87.

42. Xu, L., Stevens, J., Hilton, M.B., Seaman, S., Conrads, T.P., Veenstra, T.D. 2018. COX-2 Inhibition Potentiates AntiAngiogenic Cancer Therapy and Prevents Metastasis in Preclinical Models. Sci Transl Med. 6(242):1-24.

43. Yuan, D., Xia, M., Meng, G., Xu, C., Song, Y., Wei, J. 2015. Anti-Angiogenic Efficacy of 5'-Triphosphate SiRNA Combining VEGF Silencing and RIG-I Activation in NSCLCs. Oncotarget. 6(30):29664-29674.

44. Zhang, J. 2014. Intratumor Heterogeneity in Localized Lung Adenocarcinomas Delineated by Multiregion Sequencing. Science. 346: 256-259.

45. Zhang, Z., dkk. 2010. Conjugation Folic Acid to Gold Nanoparticle Through Glutathione for Targeting and Detecting Cancer Cells. Bioorganic Med Chem. 18(15):5528-5534. 